\title{
A Flexible Region of Interest Extraction Algorithm with Adaptive Threshold for 3-D Synthetic Aperture Radar Images
}

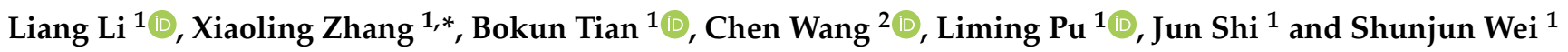 \\ 1 School of Information and Communication Engineering, University of Electronic Science and Technology \\ of China, No. 2006, Xiyuan Ave., West Hi-Tech Zone, Chengdu 611731, China; liliang@std.uestc.edu.cn (L.L.); \\ tianbokun@std.uestc.edu.cn (B.T.); puliming@std.uestc.edu.cn (L.P.); shijun@uestc.edu.cn (J.S.); \\ weishunjun@uestc.edu.cn (S.W.) \\ 2 School of Electronic and Information Engineering, Suzhou University of Science and Technology, No. 99, \\ Xuefu Road, Huqiu Zone, Suzhou 215009, China; chenwang@mail.usts.edu.cn \\ * Correspondence: xlzhang@uestc.edu.cn
}

check for

updates

Citation: Li, L.; Zhang, X.; Tian, B.; Wang, C.; Pu, L.; Shi, J.; Wei, S. A Flexible Region of Interest Extraction Algorithm with Adaptive Threshold for 3-D Synthetic Aperture Radar Images. Remote Sens. 2021, 13, 4308 https://doi.org/10.3390/rs13214308

Academic Editors: Przemysław

Kupidura and Joanna

Pluto-Kossakowska

Received: 27 September 2021

Accepted: 14 October 2021

Published: 26 October 2021

Publisher's Note: MDPI stays neutral with regard to jurisdictional claims in published maps and institutional affiliations.

Copyright: (c) 2021 by the authors. Licensee MDPI, Basel, Switzerland. This article is an open access article distributed under the terms and conditions of the Creative Commons Attribution (CC BY) license (https:/ / creativecommons.org/licenses/by/ $4.0 /$ )
Abstract: Most of the existing image segmentation methods have a strong anti-noise ability but are susceptible to the interference in the background, so they are not suitable for 3-D synthetic aperture radar (SAR) image target extraction. Region of interest (ROI) extraction can improve the anti-interference ability of the image segmentation methods. However, the existing ROI extraction method uses the same threshold to process all the images in the data set. This threshold is not optimal for each image. Designed for 3-D SAR image target extraction, we propose an ROI extraction algorithm with adaptive threshold (REAT) to enhance the anti-interference ability of the existing image segmentation methods. The required thresholds in the proposed algorithm are adaptively obtained by the mapping of the image features. Moreover, the proposed algorithm can easily be applied to existing image segmentation methods. The experiments demonstrate that the proposed algorithm significantly enhances the anti-interference ability and computational efficiency of the image segmentation methods. Compared with the existing ROI extraction algorithm, the proposed algorithm improves the dice similarity coefficient by $6.4 \%$.

Keywords: synthetic aperture radar (SAR); image segmentation; target extraction; 3-D; saliency detection

\section{Introduction}

Recently, near-field 3-D synthetic aperture radar (SAR) imaging and its applications have become an important research direction [1-3]. When SAR imaging results are displayed in 3-D form, the occlusion caused by the background (the sidelobes, noise, and interference) makes the main lobe difficult to observe. The target extraction of a 3-D SAR image is an efficient algorithm for eliminating the influence of the background. Scholars are committed to investigating the target extraction algorithm with high accuracy and computational efficiency. For target extraction, the image segmentation method is usually utilized to divide the image into several categories, and then the target is determined through certain judgment conditions.

In computer vision, medicine, and other fields, image segmentation methods based on fuzzy C-means (FCM) and hidden Markov random fields (HMRF) are widely used due to their good performance. Recently, researchers have been working hard to improve the performance of these two types of algorithms [4-8]. One of the main means of improvement is the introduction of information into neighboring voxels to improve the anti-noise ability [9-11].

We try to use general segmentation algorithms to complete the target extraction of 3-D SAR images. However, when these algorithms are applied to 3-D SAR images, it is difficult for them to avoid the influence of interference whose intensity is very close to the target. As a result, some strong interference voxels are incorrectly divided into the target 
voxels. In addition, some segmentation algorithms tend to use more complex objective functions to obtain better anti-noise ability. And these algorithms are usually designed for the processing of 2-D images. When these algorithms are applied to 3-D images, the complex objective functions make their computational efficiency unacceptable. Therefore, most of the algorithms that exist are not suitable for direct application to the segmentation of 3-D SAR images.

A 3-D SAR image has sparsity [12], which means that the number of background voxels in a 3-D SAR image is far higher than that of target voxels. We consider the region where the target is located as the region of interest (ROI). If one algorithm can be applied to remove the background area with the interference and extract ROI, then the highperformance image segmentation algorithm only needs to divide the voxels in ROI. This not only improves the anti-interference ability but also the computational efficiency.

The region-adaptive morphological reconstruction fuzzy C-means (R-AMRFCM) algorithm [13] is an ROI-based algorithm specifically designed for 3D SAR image target extraction. It utilizes the anisotropic diffusion method [14] to smooth the image, the 3-D Kirsch method to calculate the gradient magnitude, and the hysteresis threshold method to extract target edges. The minimum bounding polyhedrons of the target edges are regarded as the ROI. The adaptive morphological reconstruction fuzzy C-means algorithm was then used to divide the target voxels and the background voxels in ROI. However, R-AMRFCM has two disadvantages:

1. The gradient threshold in the anisotropic diffusion filter and the two thresholds in the hysteresis threshold method have to be adjusted manually. The adjustment process is experience-dependent and time-consuming;

2. The target extraction of different images in one data set is achieved by the same thresholds. These thresholds are not optimal for each image.

In this paper, we will focus on designing an ROI-based preprocessing algorithm which can obtain the required thresholds adaptively in order to make general segmentation algorithms suitable for 3-D SAR image target extraction. Image features can reflect the unique information contained in each image, and different images have different features. If a mapping relationship can be established between the required thresholds and image features, the thresholds can be calculated adaptively. Thereby, we aim to find a way to achieve ROI extraction with an adaptive threshold.

There are some statistical models that fit the distribution of gray levels in SAR images; these include Gamma distribution, Gamma mixture distribution (GMD), generalized Gamma distribution (GGD), etc. [15-22]. The gray levels of the 3-D SAR images that we processed $[13,23,24]$ follow Gamma distribution. Furthermore, the algorithm proposed in this paper is based on this hypothesis. The histogram is an important feature for SAR images. Researchers have achieved a variety of SAR image applications through histograms [25-28]. For example, Qin et al. proposed a classification algorithm and a segmentation algorithm for SAR images based on GGD [29,30]. Pappas et al. proposed a framework to extract a river area from a SAR image based on GGD [31]. Xiong et al. presented a change detection measure based on the statistical distribution of SAR intensity images [32]. Therefore, we also utilize the histogram to achieve an ROI extraction with an adaptive threshold.

In this paper, an ROI extraction algorithm with adaptive threshold (REAT) is proposed to improve the anti-interference ability and the computational efficiency of existing segmentation algorithms. Firstly, a fast saliency detection algorithm is applied to enhance the 3-D SAR image [33]. Secondly, the features of the original image and the enhanced image (the image mean, the histogram, etc.) are obtained. Based on the histograms of the original image and the enhanced image, the Kullback-Leibler distance (KLD) is utilized to evaluate the difference between these two images. Otsu's method [34] is used to calculate the reference thresholds. Subsequently, the gradient threshold in the anisotropic diffusion method is calculated with the histogram and the image mean. Two thresholds in the Canny edge detection method [35] are calculated with the use of the reference thresholds and the 
KLD. Then, the anisotropic diffusion method is employed to smooth the image, and the Canny edge detection method is applied to extract the target edges. Finally, the minimum bounding cuboids of the target edges are considered as the core region, and the boundary of the core region is expanded outward to form a buffer area. The core region and the buffer area constitute a complete ROI. Moreover, the expansion reduces the loss of target regions caused by inaccurate edge extraction.

The extracted ROI can be directly treated as the input of the existing image segmentation algorithms. In this paper, a Gaussian-based hidden Markov random field and some FCM-based segmentation algorithms are utilized to divide the target voxels and the background voxels in ROI.

Our contribution is summarized as follows:

1. Designed for 3-D SAR images, we propose a preprocessing algorithm to quickly extract the region of interest. The proposed algorithm can improve the performance and efficiency of general image segmentation algorithms. Moreover, the proposed algorithm is flexible and can easily be applied to existing image segmentation methods;

2. The image features are utilized to adaptively obtain the thresholds required for ROI extraction. The thresholds are independently optimized for different images.

The rest of this paper is organized as follows. In Section 2, we review related works. In Section 4, the theory of the proposed algorithm is described. Section 5 introduces the experimental results and the performance evaluations. In Section 6, we illustrate the process of the proposed algorithm and discuss the role of the buffer area. The conclusion is given in Section 7.

\section{Related Works}

\subsection{Saliency Detection}

The saliency detection algorithm is usually used to highlight regions of interest. Therefore, we use the saliency detection algorithm to enhance the target area in the original image. Reference [33] introduces a highly efficient saliency detection algorithm with the use of the spectral residual. This algorithm divides the information contained in the image into innovation and prior knowledge, where the innovation is novelty information and the prior knowledge is redundant information. After transforming the image into a logfrequency spectrum, the prior knowledge appears as a smooth curve, and the innovation forms multiple peaks on the smooth curve. The spectral residual $\mathcal{R}(f)$ is defined as the statistical singularities which are particular to the input image. The saliency detection process is expressed as follows:

$$
\begin{gathered}
S=\mathcal{G}\left\{\mathcal{F}^{-1}[\exp (\mathcal{R}(f)+\mathcal{P}(I))]^{2}\right\}, \\
\mathcal{R}(f)=\mathcal{L}(f)-\mathcal{M}[\mathcal{L}(f)], \\
\mathcal{L}(f)=\log (\mathcal{A}(I)),
\end{gathered}
$$

where $\mathcal{A}(I)$ denotes the amplitude of the image $I$ after Fourier transform, $\mathcal{P}(I)$ denotes the phase of the image $I$ after Fourier transform, $\mathcal{M}$ denotes mean filtering, $\mathcal{F}^{-1}[\cdot]$ denotes inverse Fourier transform, $\mathcal{G}\{\cdot\}$ denotes Gaussian filtering. The saliency map $S$ indicates the regions of interest in the image.

\subsection{Gaussian-Based Hidden Markov Random Field}

After the ROI extraction by the proposed algorithm, an image segmentation method is needed to divide the target voxels and the background voxels in the ROI. We use a classic method, the Gaussian-based hidden Markov random field (G-HMRF), to achieve the segmentation. 
Assuming that the original image contains $N$ voxels, $h_{n}$ denotes the gray level of the $n$th voxel, and the set of gray levels is $\mathcal{H}=\left\{h_{1}, \ldots, h_{N}\right\}$. Each voxel corresponds to a label $v_{n}, \mathcal{V}=\left\{v_{1}, \ldots, v_{n}, \ldots, v_{N}\right\}, v_{n} \in[1, L]$, with $L$ as the number of categories. For the hidden Markov random field, $\mathcal{H}$ is the observed field, which is known. $\mathcal{V}$ is a hidden random field. The purpose of the hidden Markov random field is to estimate the hidden random field $\mathcal{V}$ based on the observed field $\mathcal{H}$.

The maximum a posterior (MAP) criterion finds the best label $\mathcal{V}^{*}$ by maximizing the posterior probability, which is usually used to optimize G-HMRF. Assuming that $\hat{\mathcal{V}}$ is the estimate of the label $\mathcal{V}^{*}$,

$$
\hat{\mathcal{V}}=\underset{\mathcal{V}}{\operatorname{argmax}}\{P(\mathcal{V} \mid \mathcal{H}, \Theta)\}
$$

where $\Theta=\left\{\theta_{l} \mid l \in L\right\}$ denotes the parameter set. The Bayesian criterion is expressed as:

$$
P(\mathcal{V} \mid \mathcal{H}, \Theta)=\frac{P(\mathcal{H} \mid \mathcal{V}, \Theta) P(\mathcal{V})}{P(\mathcal{H})} .
$$

where $P(\mathcal{H})$ is a constant, which can be ignored in the calculation process. According to the simplified Bayesian criterion, we have the following:

$$
P(\mathcal{V} \mid \mathcal{H}, \Theta)=P(\mathcal{H} \mid \mathcal{V}, \Theta) P(\mathcal{V}),
$$

Equation (4) is rewritten as:

$$
\hat{\mathcal{V}}=\underset{\mathcal{V}}{\operatorname{argmax}}\{P(\mathcal{H} \mid \mathcal{V}, \Theta) P(\mathcal{V})\} .
$$

The Hammersly-Clifford principle indicates that the Markov random field and Gibbs distributions are identical [36]. Therefore, maximizing the posterior probability is equivalent to minimizing the energy function,

$$
\hat{\mathcal{V}}=\underset{\mathcal{V} \in \chi}{\operatorname{argmin}}\{U(\mathcal{H} \mid \mathcal{V}, \Theta)+U(\mathcal{V})\},
$$

where $\chi$ denotes all possible configurations of labels. The unitary potential function is expressed as follows [37]:

$$
\begin{aligned}
U(\mathcal{H} \mid \mathcal{V}, \Theta) & =\sum_{n=1}^{N} U\left(h_{n} \mid v_{n}, \Theta\right) \\
& =\sum_{n=1}^{N}\left[\frac{\left(h_{n}-\mu_{v_{n}}\right)^{2}}{2 \sigma_{v_{n}}^{2}}+\ln \sigma_{v_{n}}\right],
\end{aligned}
$$

and the pairwise potential function is expressed as [37]:

$$
\begin{gathered}
U(\mathcal{V})=\sum_{c \in C} V_{c}\left(v_{i}, v_{j}\right), \\
V_{c}\left(v_{i}, v_{j}\right)=\left\{\begin{array}{ll}
B & \text { if } v_{i} \neq v_{j} \\
0 & \text { if } v_{i}=v_{j}
\end{array},\right.
\end{gathered}
$$

where $V_{c}\left(v_{i}, v_{j}\right)$ denotes the clique potential and $C$ denotes the set of all possible cliques. Based on experience, the variable coefficient $B$ is set to $1 / 2$ in this paper.

After estimating $\hat{\mathcal{V}}$ through MAP, the parameter set $\Theta$ needs to be estimated. This step is usually achieved by the expectation maximization algorithm (EM). The image segmentation process through G-HMRF is described as follows:

1. K-means or fuzzy C-means is used to initialize the label $\hat{\mathcal{V}}^{(0)}$. According to $\hat{\mathcal{V}}^{(0)}$ and the set of gray levels $\mathcal{H}, \mathrm{EM}$ is applied to initialize the parameter set $\Theta^{(0)}$; 
2. MAP is utilized to estimate the label $\hat{\mathcal{V}}^{(t+1)}$ :

$$
\hat{\mathcal{V}}^{(t+1)}=\underset{\mathcal{V}^{(t)} \in \chi}{\operatorname{argmin}}\left\{U\left(\mathcal{H} \mid \mathcal{V}^{(t)}, \Theta^{(t)}\right)+U\left(\mathcal{V}^{(t)}\right)\right\}
$$

3. According to the label $\hat{\mathcal{V}}^{(t+1)}$ and the set of gray levels $\mathcal{H}$, EM is used to estimate the new parameter set $\Theta^{(t+1)}$;

4. After $z$ iterations of step 2 and step 3 , the division $\hat{\mathcal{V}}^{z}$ of each voxel in the image is obtained.

\section{Background}

The 3-D SAR imaging system for security inspection usually consists of three main parts: a virtual array, a radio frequency system (RFS) for transmitting signals and collecting echoes, and a data processing platform (DPP) for storing the digital echo data and imaging. And the framework of the 3-D SAR imaging system for security inspection is shown in Figure 1. The SAR system uses wideband signals and matched filtering to obtain range resolution. In order to obtain the resolution of the other two dimensions (cross-track direction and along-track direction), a virtual array is generated on a plane perpendicular to the distance direction. For the security inspection SAR imaging system, a 1-D real antenna array is usually used to generate a virtual array. This mode takes into account the cost and scanning time.

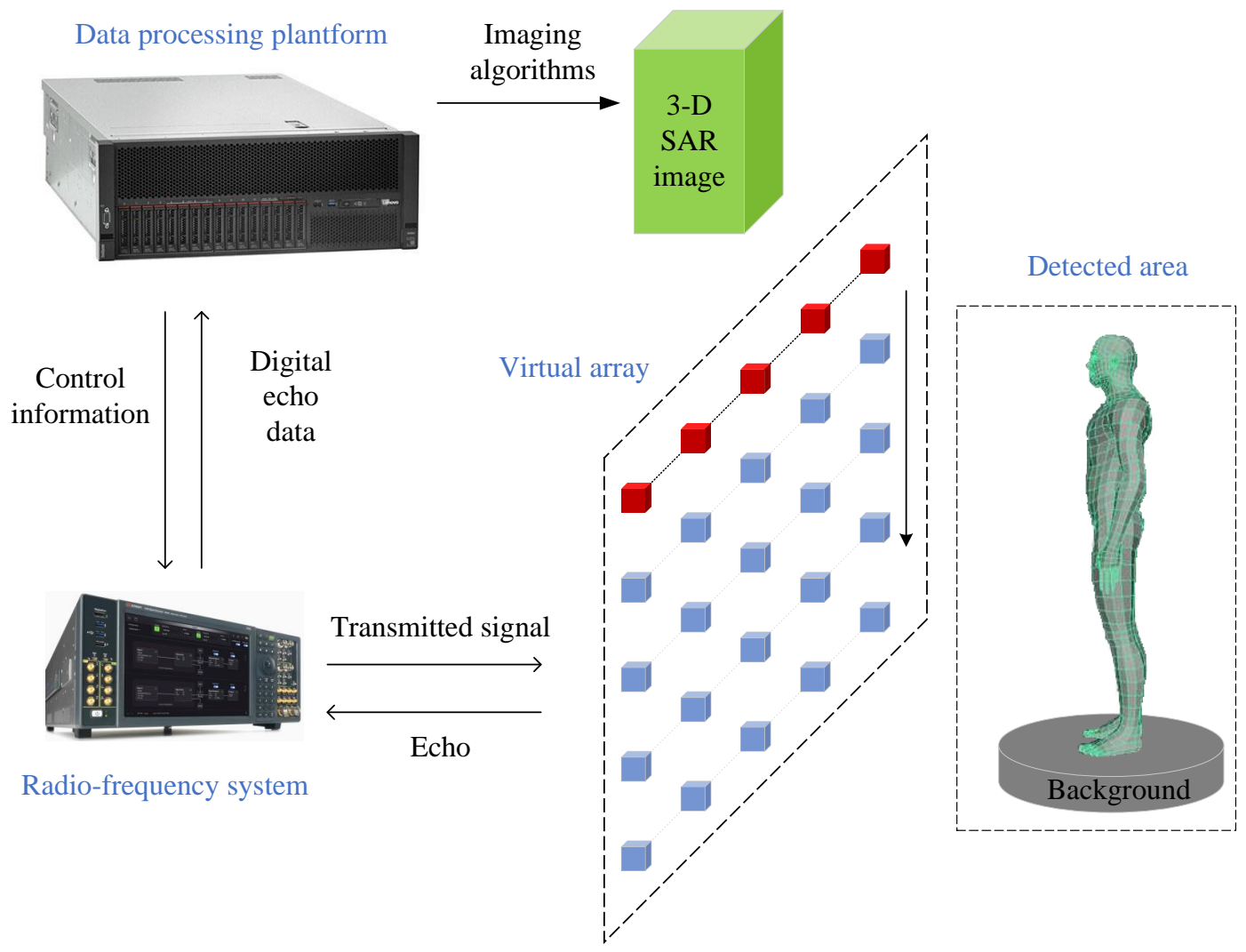

Figure 1. The framework of the 3-D SAR imaging system for security inspection. It usually consists of three main parts: a virtual array, a radio-frequency system, and a data processing platform.

The DPP sends the control information to the RFS. Then, the RFS transmits signals and receives echoes through antennas. After the analog-to-digital conversion, the digital echo data is transmitted from the RFS to the DPP. Finally, the DPP stores the digital echo data and achieves 3-D SAR imaging. References $[23,24]$ show two different near-field 3-D SAR imaging systems. 
The 3-D SAR image can be divided into two parts: target and background. There are some high-intensity interferences in the background, and the intensity of these interferences is almost equal to the intensity of the weak target. For general image segmentation algorithms, it is difficult to distinguish between weak targets and strong interferences based on intensity alone. Reference [13] shows that the edge gradient intensity of the target is usually stronger than that of the interference. Therefore, the target and the interference can be distinguished by the difference in edge gradient intensity. This paper designs an ROI extraction algorithm based on this idea.

\section{Method}

Influenced by interference, general image segmentation algorithms are not suitable for the target extraction of 3-D SAR images. Therefore, we propose an ROI extraction algorithm with adaptive threshold (REAT) to enhance the anti-interference ability and computational efficiency of general image segmentation algorithms. The input of REAT is a 3-D gray scale voxel image, and its output is one or more small-sized 3-D gray scale images (regions of interest). For near-field 3-D SAR images, voxels with higher gray levels tend to be judged as target voxels. When designing REAT, the area gathered by voxels with higher gray levels is judged as the region of interest. In this section, we will elaborate on the inspiration and theory of the proposed algorithm.

\subsection{Overview}

The proposed algorithm consists of 4 main steps, and the structure is shown in Figure 2. Firstly, the saliency map of the original image is calculated by the spectral residual method. Based on the saliency map, the enhancement matrix is generated. The saliency-enhanced image is calculated by the Hadamard product of the original image and the enhancement matrix. Secondly, the mean value of the enhanced image, the histogram of the enhanced image, and the histogram of the original image are calculated. In addition, two reference thresholds are calculated by Otsu's method based on the histogram of the original. After calculating the probability distribution function of the original image and the enhanced image with the histogram, the Kullback-Leibler distance between these two images is obtained. Then, the gradient threshold of the anisotropic diffusion algorithm and the thresholds of the Canny edge detection algorithm are calculated by two map functions with the above-mentioned results. Finally, after smoothing the enhanced image with the anisotropic diffusion algorithm, the Canny algorithm is used to detect the target edges. The minimum bounding cuboids of the target edges are regarded as the core region. After expanding the boundary of the cuboids outwards, the expanded area is then considered the buffer area. The core region and buffer area constitute the complete ROI.

We introduce the proposed algorithm in detail in the following subsections.

\subsection{Image Enhanced by Saliency Detection}

Assuming that the matrix of the gray levels of the original image is $\mathbf{I}, I(x, y, z)$ is the element in $\mathbf{I}, x \in\left[1, N_{x}\right], y \in\left[1, N_{y}\right], z \in\left[1, N_{z}\right]$. The set of gray levels of all voxels is $\mathcal{H}=\left\{h_{1}, \ldots, h_{n}, \ldots, h_{N}\right\}, N=N_{x} \times N_{y} \times N_{z}$. The spectral residual method is applied to calculate the saliency map $\mathbf{S}$ of $\mathbf{I}$. $\tilde{\mathbf{S}}$ is the gray-level normalized result of $\mathbf{S}$. Then, the enhancement matrix is constructed as follows:

$$
\mathbf{E}=(1-p) \cdot \tilde{\mathbf{A}}+p \cdot \tilde{\mathbf{S}},
$$

where $p \in(0,1)$ denotes the gain, $\tilde{\mathbf{A}} \in \mathbb{Z}^{N_{x} \times N_{y} \times N_{z}}$ denotes a matrix whose elements are all 1. The enhanced image $\dot{\mathbf{S}}$ is expressed as:

$$
\dot{\mathbf{S}}=\mathbf{E} \odot \mathbf{I},
$$

where $\odot$ denotes the Hadamard product. Image saliency enhancement reduces the intensity of the background area. We will illustrate this in Section 6.1. 


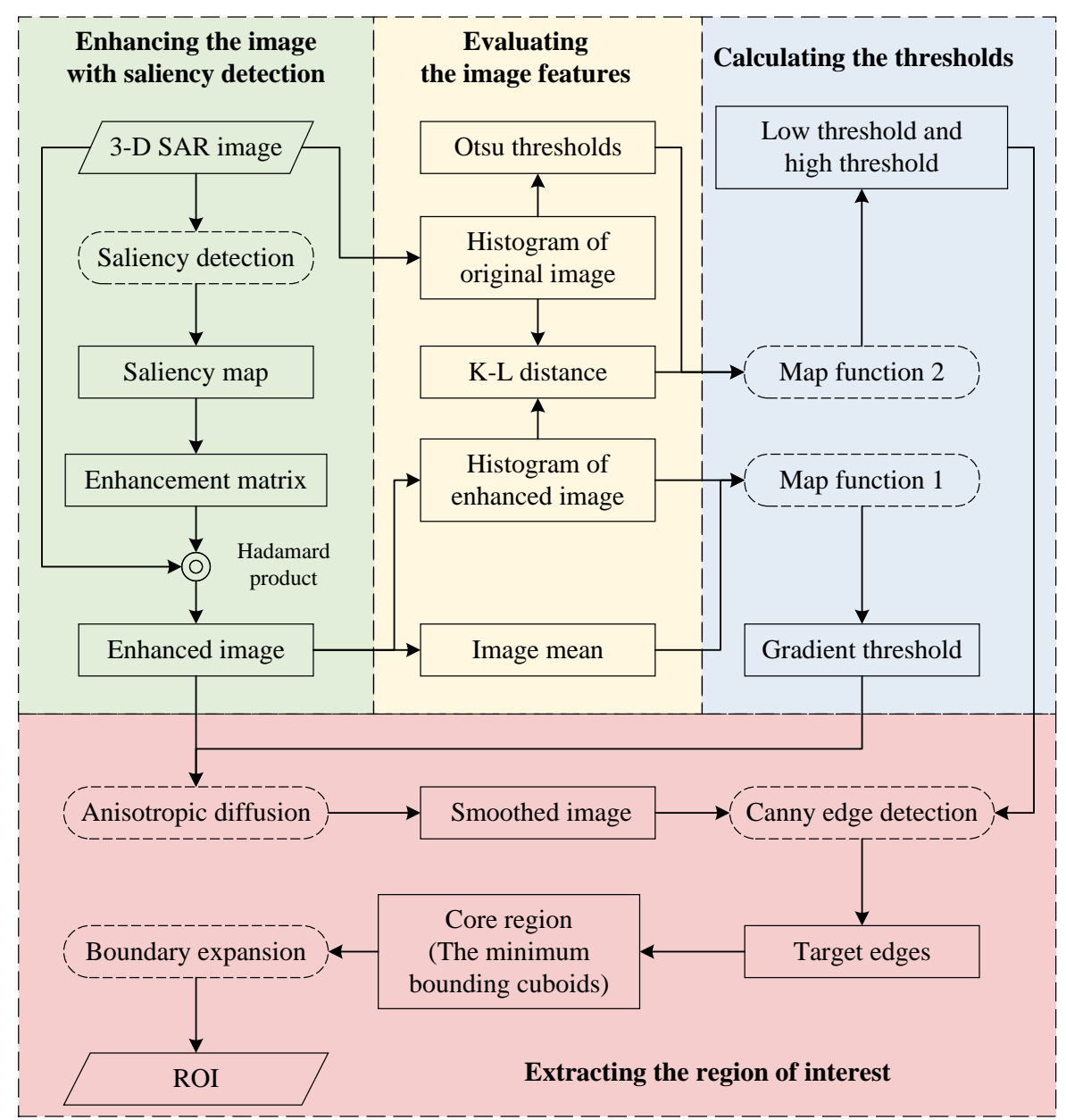

Figure 2. The structure of the proposed algorithm. It consists of 4 main steps: enhancing the image with saliency detection, evaluating image features, calculating the thresholds, and extracting the region of interest.

\subsection{The Calculation of the Gradient Threshold}

In this paper, assume that a 3-D SAR image follows the Gamma distribution. The schematic of a 3-D SAR image histogram is shown in Figure 3. For near-field SAR detection, the target usually has high intensity. Due to the sparsity of 3-D SAR images, the number of background voxels with low gray levels is much higher than the number of target voxels with high gray levels. Thus, there is a serious imbalance between the number of target voxels and the number of background voxels. In addition, the histogram is not a smooth curve, and there are many small peaks on the histogram.

The gradient threshold in the anisotropic diffusion algorithm depends on the noise level and edge strength [14]. In this paper, the gradient threshold is regarded as the boundary between the target and the background. According to the histogram, the huge peak in the low gray-level range is almost contributed by the background. If the gray level with the largest number of voxels is regarded as the median of the background intensity, two times the median of the background intensity can be roughly regarded as the boundary between target and background. However, this boundary is unstable and susceptible to small peaks. To improve the stability of the boundary, the mean value of $N_{A D}$ gray levels with the largest number of voxels is taken as the median of the background intensity. The larger the $N_{A D}$, the more stable the median of the background intensity. However, because the histogram of the Gamma distribution is asymmetric, too large an $N_{A D}$ will cause the offset of the median of the background intensity. Empirically, $N_{A D}$ is set to 5 . 


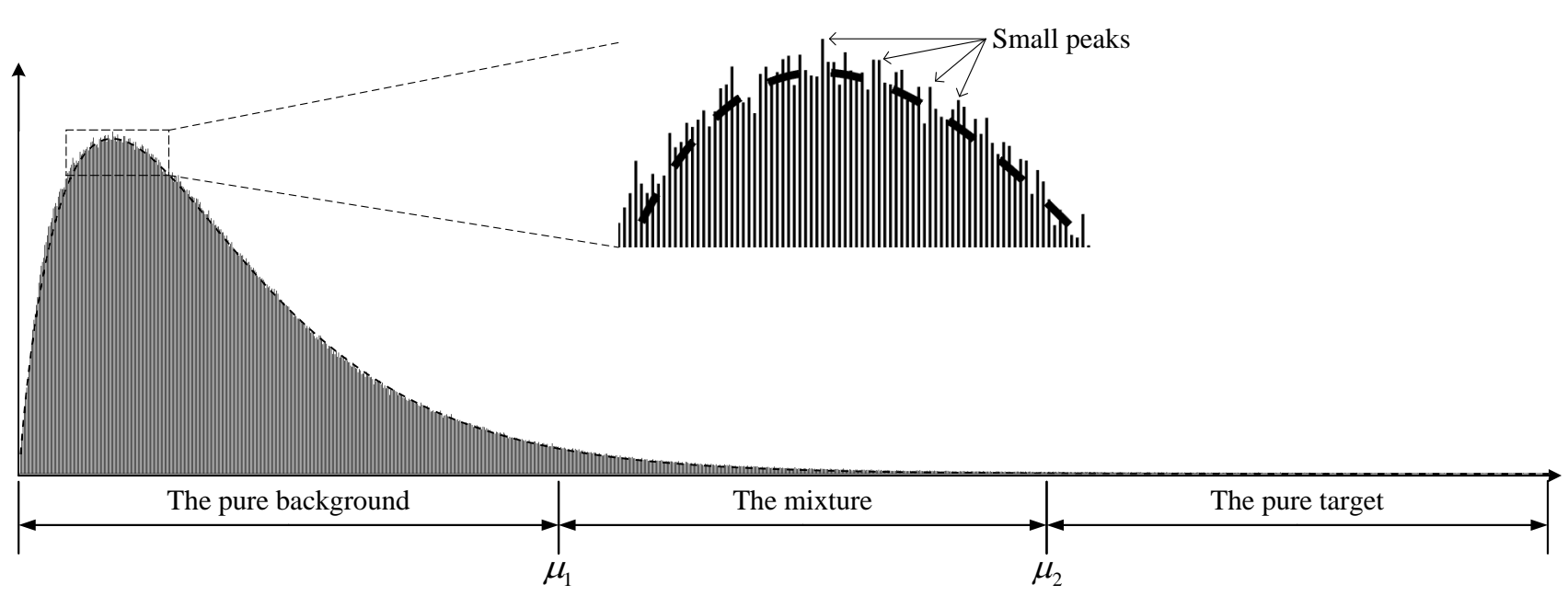

Figure 3. The histogram of a 3-D SAR image. It is divided into three areas: the pure background, the mixture of the target and the background, and the pure target.

The calculation of the median of the background intensity only considers the image background information and ignores the target information. We believe that the gradient threshold should be calculated after a complete evaluation of the image. Therefore, the image mean that includes both background information and target information is introduced to correct the threshold calculated before.

The set of gray levels of the enhanced image is $\dot{\mathcal{H}}=\left\{\dot{h}_{1}, \dot{h}_{n}, \ldots, \dot{h}_{N}\right\}$, and the mean value of the image is $\dot{\mathcal{H}}$ is $\bar{h}=\frac{1}{N} \sum_{n=1}^{N} \dot{h}_{n}$. The histograms of the original image and the enhanced image are $\Gamma=\left\{\gamma_{1}, \gamma_{2}, \ldots, \gamma_{q}\right\}$ and $\dot{\Gamma}=\left\{\dot{\gamma}_{1}, \dot{\gamma}_{2}, \ldots, \dot{\gamma}_{q}\right\}$, respectively; $q \in[1, Q]$. Both histograms have the same gray level $\mathcal{R}=\left\{r_{1}, r_{2}, \ldots, r_{q}\right\}$. The histogram of the enhanced image is sorted from largest to smallest. The sorted histogram is $\left\{\dot{\gamma}_{1}^{\prime}, \dot{\gamma}_{2}^{\prime}, \ldots, \dot{\gamma}_{q}^{\prime}\right\}$, where the gray level corresponding to $\left\{\dot{\gamma}_{1}^{\prime}, \dot{\gamma}_{2}^{\prime}, \dot{\gamma}_{3}^{\prime}, \dot{\gamma}_{4}^{\prime}, \dot{\gamma}_{5}^{\prime}\right\}$ is $\left\{\dot{r}_{1}^{\prime}, \dot{r}_{2}^{\prime}, \dot{r}_{3}^{\prime}, \dot{r}_{4}^{\prime}, \dot{r}_{5}^{\prime}\right\}$. Hence, the median of the background intensity is $\bar{r}=\frac{1}{5} \sum_{q=1}^{5} \dot{r}_{q}^{\prime}$. The gradient threshold $\kappa$ is calculated with the following map function:

$$
\kappa=\frac{2 \bar{r}+\bar{h}}{2} .
$$

So far, we have obtained the gradient threshold $\kappa$ for the anisotropic diffusion algorithm.

\subsection{Canny Edge Detection and the Calculation of Two Thresholds}

According to the distribution of voxels in the histogram, it is inferred that there is a threshold $\mu_{1}$ so that the voxels whose gray level is in the range of $\left[0, \mu_{1}\right]$ are part of the background. Similarly, there is a threshold $\mu_{2}$ so that the voxels whose gray level is higher than $\mu_{2}$ are targets. Thus, these two thresholds divide the histogram into three intervals: the pure background, the mixture of the target and the background, and the pure target.

According to the threshold $\mu_{1}$, the histogram is divided into two regions. The voxels belonging to the pure background are directly discarded. The voxels belonging to the mixture and the pure target are extracted and considered as the ROI. This allows for a large number of background voxels to be removed.

There is some strong interference in the 3-D SAR image. These interference voxels are usually distributed in the pure background and the mixture. If the ROI and the background are roughly divided by the threshold $\mu_{1}$, the interference voxels in the mixture will be divided into the ROI at the same time. In addition, these interference voxels seriously influence the segmentation result. Reference [13] pointed out that there is a difference between the edge gradient magnitude of the target and the interference, which 
can be used to eliminate the influence of the interference. According to this idea, the Canny edge detection method is applied to extract the target edges while eliminating the interference. The minimum bounding cuboids of the target edges after boundary expansion also forms the ROI. Among them, $\mu_{1}$ and $\mu_{2}$ correspond to the low threshold and the high threshold, respectively. In this way, ROI extraction not only removes the voxels in the pure background, but also eliminates the interference voxels in the mixture. The difficulty of image segmentation is greatly decreased.

Otsu's method [34] is an efficient histogram-based method of calculating the reference thresholds. However, the imbalance in the number of voxels between the background and the target makes the reference thresholds inaccurate. Therefore, the imbalance needs to be corrected. For the images before and after the saliency enhancement, there are differences in the background and target information between these two images. Moreover, the KullbackLeibler distance can measure these differences. Therefore, we utilize the Kullback-Leibler distance to evaluate these differences and use the evaluation result to correct the reference thresholds. The map function is used to achieve further adjustment.

Assuming that the reference thresholds calculated by Otsu's method are $\mu_{\mathrm{O} 1}$ and $\mu_{\mathrm{O} 2}$, respectively, and $\mu_{\mathrm{O} 1}<\mu_{\mathrm{O} 2}$. The probability distribution of the original image $\rho_{r}$ and the probability distribution of the enhanced image $\rho_{e}$ are calculated with the corresponding histogram, respectively:

$$
\rho_{r}\left(r_{q}\right)=\frac{\gamma_{q}}{N}, \rho_{e}\left(r_{q}\right)=\frac{\dot{\gamma}_{q}}{N}
$$

The Kullback-Leibler distance between the original image and the enhanced image is expressed as follows:

$$
\begin{gathered}
\overline{\mathcal{D}}=\frac{1}{2}\left(\mathcal{D}\left(\rho_{r} \mid \rho_{e}\right)+\mathcal{D}\left(\rho_{e} \mid \rho_{r}\right)\right), \\
\mathcal{D}\left(\rho_{r} \mid \rho_{e}\right)=\sum_{q=1}^{Q} \rho_{r}\left(r_{q}\right) \cdot \log \frac{\rho_{r}\left(r_{q}\right)}{\rho_{e}\left(r_{q}\right)}, \\
\mathcal{D}\left(\rho_{e} \mid \rho_{r}\right)=\sum_{q=1}^{Q} \rho_{e}\left(r_{q}\right) \cdot \log \frac{\rho_{e}\left(r_{q}\right)}{\rho_{r}\left(r_{q}\right)} .
\end{gathered}
$$

The thresholds after correction are expressed as follows:

$$
\mu_{D 1}=\mu_{O 1}+\overline{\mathcal{D}}, \mu_{D 2}=\mu_{O 2}+\overline{\mathcal{D}} .
$$

The range of $\mu_{\mathrm{O} 1}$ and $\mu_{\mathrm{O} 2}$ are $(0,1) . \overline{\mathcal{D}}$ is usually a small value.

If $\mu_{D 1}$ and $\mu_{D 2}$ are directly regarded as the low threshold and the high threshold, when $\mu_{D 1}$ is much smaller than $\mu_{D 2}$, some interference edges will be regarded as the target edges. Hence, the power function is introduced to narrow the gap between these two thresholds. However, the power function increases the values of $\mu_{D 1}$ and $\mu_{D 2}$ at the same time. The coefficient $\alpha$ is thus introduced to offset the negative effects of the power function. The map function is constructed as follows:

$$
\mu_{1}=\alpha \cdot\left(\mu_{D 1}\right)^{\beta}, \mu_{2}=\alpha \cdot\left(\mu_{D 2}\right)^{\beta},
$$

where $\alpha \in(0,1)$ and $\beta \in(0,1)$ are the coefficients. Figure 4 shows the relationship between these two coefficients and the map function in the range of $[0,1]$. The value with $[0,1]$ is mapped to the value with $[0, \alpha]$. The smaller the coefficient $\beta$, the smaller the gap between $\mu_{1}$ and $\mu_{2}$. In this paper, $\alpha$ and $\beta$ are set to 0.5 and $1 / 3$, respectively. 


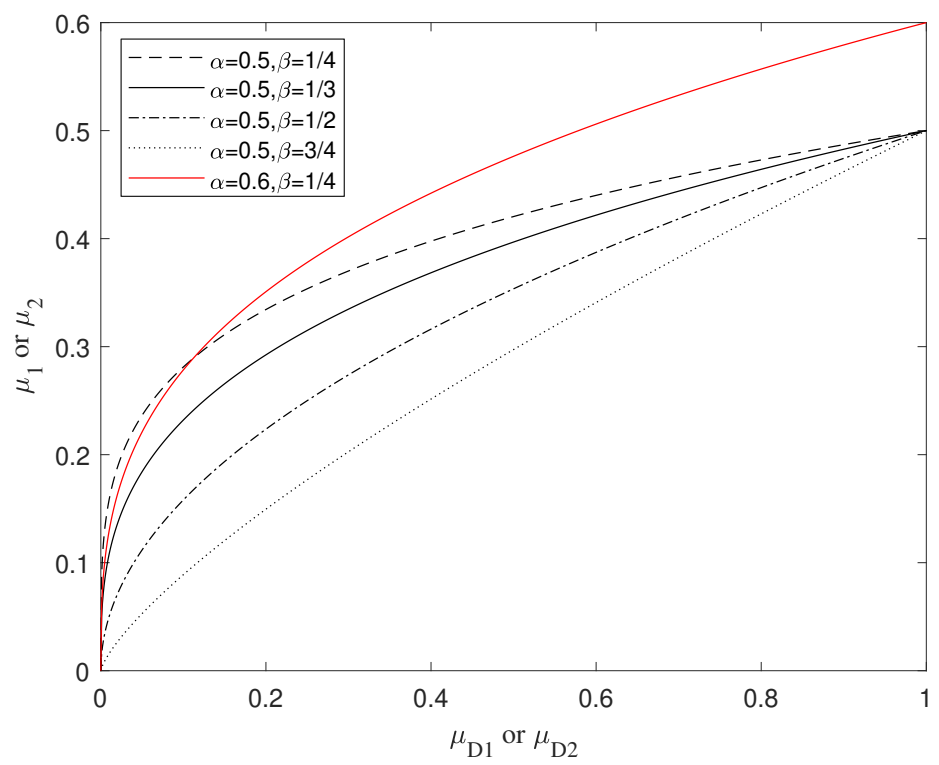

Figure 4. The relationship between the two coefficients and the map function.

\subsection{The Extraction of ROI and Target}

The thresholds required by the anisotropic diffusion method and the Canny edge detection algorithm have been calculated. After smoothing the enhanced image with the anisotropic diffusion method, the Canny algorithm is applied to extract the target edges. Thus, several regions formed by the minimum bounding cuboids of the target edges are determined, and the set of these regions is regarded as the core region. The inaccurate edge detection causes the permanent loss of part of the target region. Therefore, we expand the boundary of the core region outward to the $\left(\tau_{x}, \tau_{y}, \tau_{z}\right)$ voxels. The expanded area is named the buffer area. The core region and the buffer area form the complete ROI $\Omega=\left\{\omega_{1}, \omega_{2}, \ldots, \omega_{w}\right\}, w \in[1, W]$. The next step is to accurately divide the voxels in each region $\omega_{w}$ through an image segmentation method.

Each region $\omega_{w}$ is composed of target voxels and background voxels, $\omega_{w}=\omega_{w}^{t} \cup \omega_{w}^{b}$, where $\omega_{w}^{t}$ denotes the set of target voxels in the $w$ th region, and $\omega_{w}^{b}$ denotes the set of background voxels in the wth region. G-HMRF is used to achieve the division of voxels in each region $\omega_{w}$. The category containing the highest gray-level voxels is marked as the target, and the other category is marked as the background. Thereby, the union of all target voxel sets $\Omega^{t}=\omega_{1}^{t} \cup \omega_{2}^{t} \cup \cdots \cup \omega_{w}^{t}$ is extracted. To decrease over-segmentation, the voxels whose gray level is less than $\mu_{1} \cdot r_{Q}$ are regarded as background and removed. To improve the accuracy, the $\psi$ times upsampling is performed on each region before image segmentation, and the region size is restored after the image segmentation is completed.

\section{Experiments}

\subsection{Evaluation Criterion}

Accuracy, precision, recall, and the dice similarity coefficient (DSC) were used to evaluate the performance of the target extraction algorithm. $R_{a}$ denotes the whole image. $R_{\text {seg }}$ denotes the extracted target region. $R_{g t}$ denotes the ground truth.

Accuracy represents the ratio of the number of voxels correctly classified to the number of total voxels in the image:

$$
\text { Accuracy }=\frac{\left|R_{\text {seg }} \cap R_{g t}\right|+\left|R_{a}-\left(R_{g t} \cup R_{\text {seg }}\right)\right|}{\left|R_{a}\right|} .
$$

Precision represents the ratio of the number of correctly extracted target voxels to the number of all extracted target voxels: 


$$
\text { Precision }=\frac{\left|R_{\text {seg }} \cap R_{g t}\right|}{\left|R_{\text {seg }}\right|} .
$$

Recall represents the ratio of the number of correctly extracted target voxels to the number of real target voxels:

$$
\text { Recall }=\frac{\left|R_{\text {seg }} \cap R_{g t}\right|}{\left|R_{g t}\right|} .
$$

The dice similarity coefficient, also known as the $F_{1}$-score, indicates the similarity between the correctly extracted target region and the real target region:

$$
D S C=\frac{2 \times\left|R_{\text {seg }} \cap R_{g t}\right|}{\left|R_{\text {seg }}\right|+\left|R_{g t}\right|} .
$$

The value range of the four criteria above is $[0,1]$. The closer the value is to 1 , the better the performance is. Among them, the DSC is the most important because of its comprehensive evaluation ability.

\subsection{Experimental Results}

We used the imaging result of the 3-D SAR security inspection system to evaluate the algorithm performance. Based on the data set in reference [13], we added several images to the data set. The new data set contains a total of 56 images, which consists of 53 images with a size of $160 \times 400 \times 40,2$ images with a size of $512 \times 512 \times 64$, and 1 image with a size of $200 \times 200 \times 41$. All experiments were performed on a workstation with an AMD Threadripper 3960X $3.8 \mathrm{GHz}$ CPU and $64 \mathrm{~GB}$ memory. The parameters of the proposed algorithm were set as follows: the gain $p=0.2$, the coefficient $\alpha=0.5$, the coefficient $\beta=1 / 3, \tau_{x}=6, \tau_{y}=6, \tau_{z}=0$, the upsampling rate $\psi=2$.

To verify the enhancement ability of the proposed algorithm to the image segmentation method, we used the proposed algorithm to enhance G-HMRF [37], FRFCM [38], and RSSFCA [39]. The enhanced algorithms were named REAT-G-HMRF, REAT-FRFCM, and REAT-RSSFCA, respectively. R-AMRFCM [13] is a state-of-the-art algorithm for 3-D SAR image target extraction. To verify the performance of the proposed algorithm, the ROI extraction algorithm in R-AMRFCM was replaced with the proposed algorithm. The algorithm after replacement was called REAT-AMRFCM.

The target extraction results of the four images in the data set are shown in Figure 5. For a clear display, we converted the 3-D image into a 2-D image with the maximum projection along the $\mathrm{Z}$ direction. The original image is in the leftmost column, and the ground truth is next to the original image. The results of the original algorithms are marked in white, the results of the algorithms enhanced by the REAT are marked in yellow, and the results of the state-of-the-art algorithms are marked in red.

As shown in Figure 5, the target extraction results of the three original algorithms (G-HMRF, FRFCM, and RSSFCA) contain a lot of background voxels. In contrast, the extraction results of the three enhanced algorithms (REAT-G-HMRF, REAT-FRFCM, and REAT-RSSFCA) almost do not contain background voxels. Additionally, as shown in Figure 5d, the result of REAT-AMRFCM contains fewer background voxels than that of R-AMRFCM. Therefore, the proposed algorithm enhances the anti-interference ability of the existing image segmentation methods and has a stronger anti-interference ability than the state-of-the-art algorithm. 


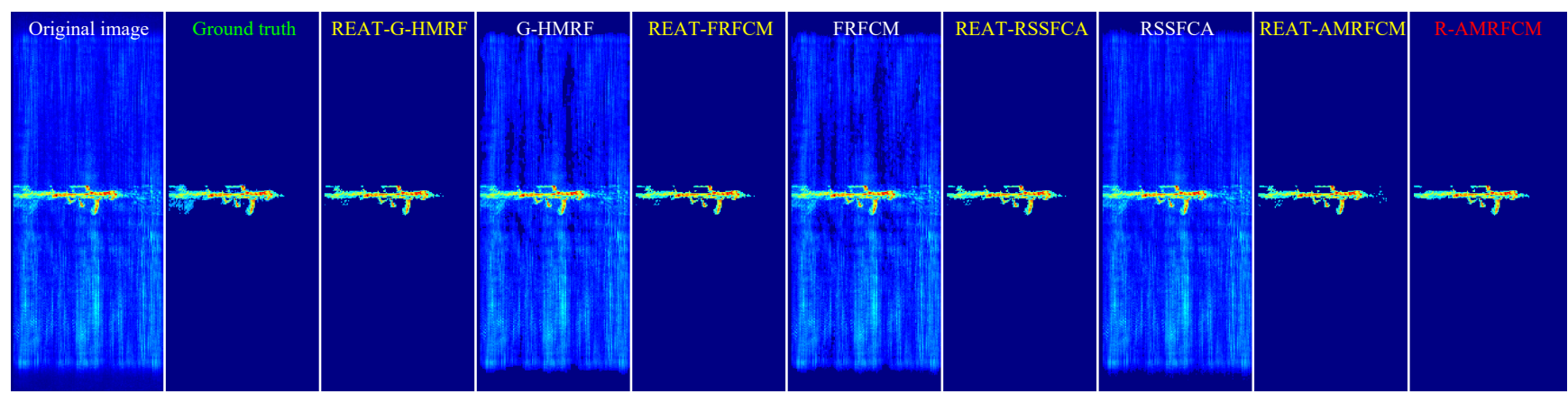

(a)

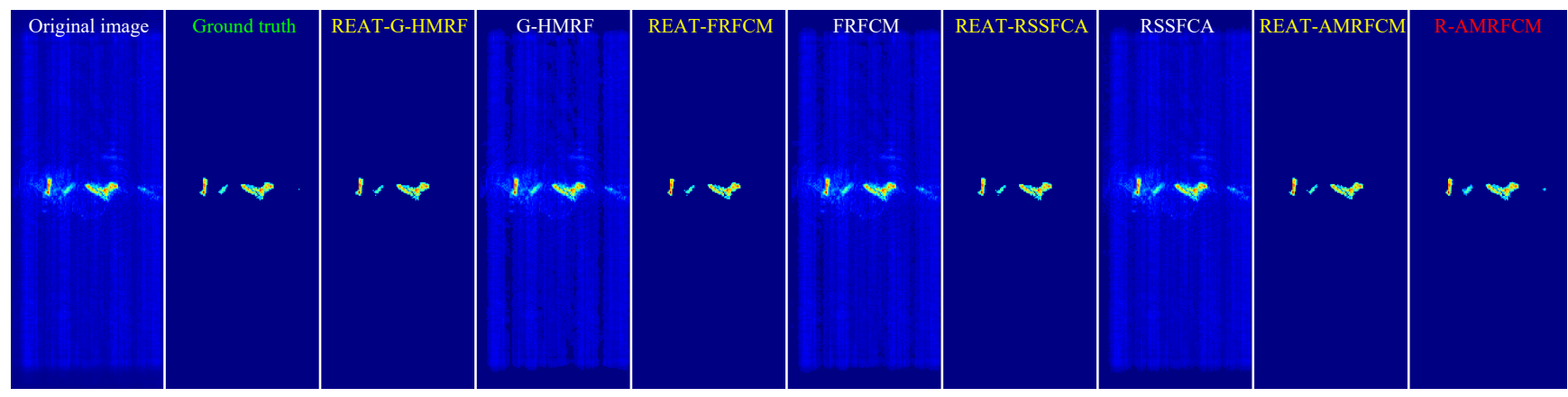

(b)

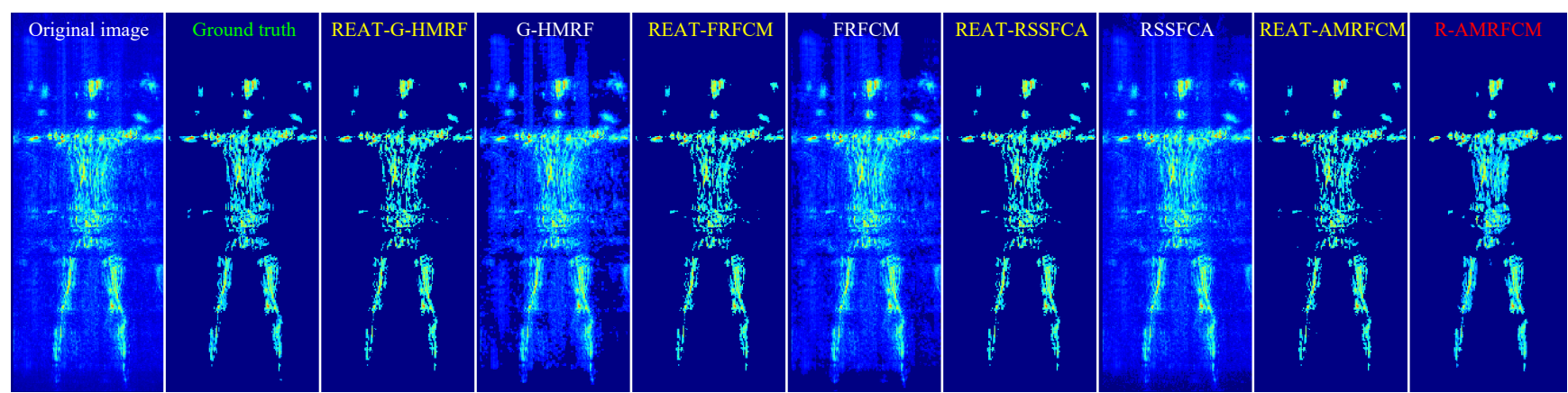

(c)

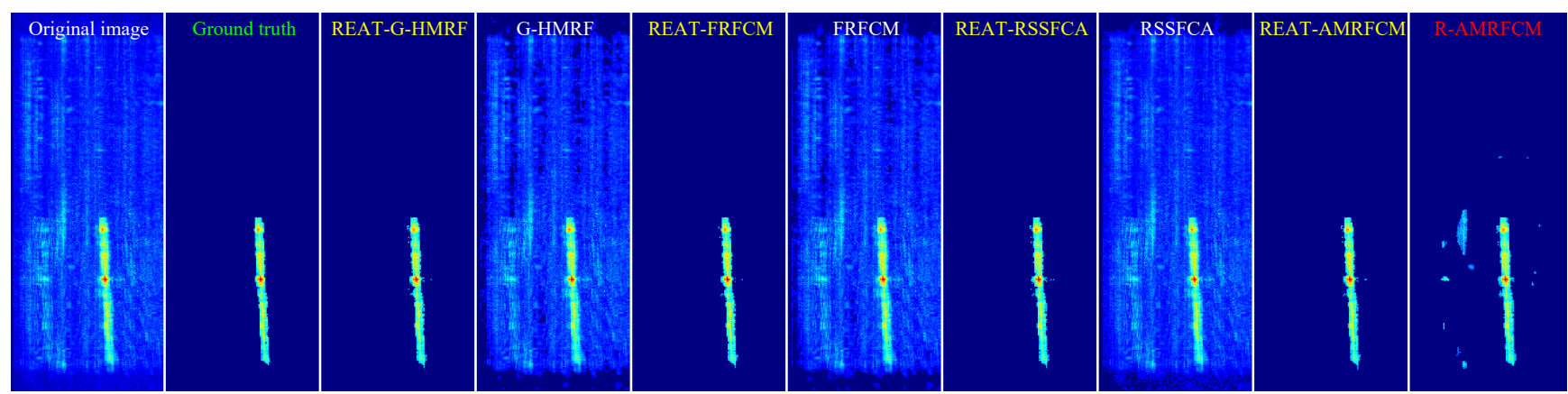

(d)

Figure 5. The original image, the ground truth, and the target extraction results of 4 images in the data set. (a) Rifle. (b) Knife and pistol. (c) Human body. (d) Metal stick. 
As shown in Figure 6, there is a 3-D original image of the human body, a 3-D ground truth, and 3-D extraction results of 8 algorithms. The original image is shown in Figure 6a. Visually, the side lobes and interference in the imaging space cover the main lobe. Figure $6 \mathrm{~b}-\mathrm{d}$ are the extraction results of G-HMRF, FRFCM, and RSSFCA, respectively. The extraction results of these algorithms still contain a lot of background voxels (sidelobes and interference). As shown in Figure 6e, R-AMRFCM effectively divides the target voxels and most of the background voxels. However, some regions in the extraction result are over-segmented, and a large number of target voxels are lost in some areas. The areas where the target voxels are lost are marked by red circles. As shown in Figure $6 \mathrm{~g}-\mathrm{j}$, the enhanced segmentation algorithms achieve excellent target extraction. There is no obvious over-segmentation and lack of target regions in these extraction results.

As shown in Figure 7, the original SAR images and extraction results of 10 scenes are shown through the maximum projection. These scenes contain a rich variety of targets. For example, scene $C$ contains two tiny objects: a tomato and a tiny metal ball. Although the extraction of tiny targets in a scene full of interference is a severe test for the algorithm, the algorithms enhanced by REAT still complete the target extraction. Scene D contains the main lobe and the grating lobes of a rifle and a knife. The grating lobes are regarded as the weak targets. Among these four enhanced algorithms, REAT-AMRFCM extracts more target grating lobes than the other three algorithms. Therefore, REAT-AMRFCM is better at classifying weaker voxels as target voxels than the other three enhanced algorithms.

\subsection{Evaluation of the Performance}

Accuracy, precision, recall, and the DSC are used to evaluate the performance of these algorithms. The evaluation results of the original algorithms and the enhanced algorithms are shown in Table 1. The accuracy of these three enhanced algorithms is very close to 1. Additionally, their precision, recall, and DSC are all higher than 0.7989. Among them, REAT-FRFCM has the highest precision (0.9125), REAT-RSSFCA has the highest recall (0.8702), and REAT-G-HMRF has the highest DSC (0.8703). The accuracy, the precision, and the DSC of the enhanced algorithms are significantly higher than those of the original algorithms. The extraction results of the original methods not only contain almost all target voxels but also contain a large number of background voxels. The over-segmentation makes the original methods have a very high recall. In summary, the proposed algorithm effectively enhances the performance of the existing image segmentation methods.

Table 1. Evaluation results of the original algorithms and the enhanced algorithms. The bold number indicates the best performance under one criterion.

\begin{tabular}{cccccc}
\hline Method & ROI & Accuracy & Precision & Recall & DSC \\
\hline REAT-G-HMRF & $\checkmark$ & $\mathbf{0 . 9 9 9 4}$ & $\mathbf{0 . 8 9 2 4}$ & 0.8602 & $\mathbf{0 . 8 7 0 3}$ \\
G-HMRF & $\boldsymbol{x}$ & 0.7617 & 0.0145 & $\mathbf{1}$ & 0.0276 \\
\hline REAT-FRFCM & $\checkmark$ & $\mathbf{0 . 9 9 9 4}$ & $\mathbf{0 . 9 1 2 5}$ & 0.7989 & $\mathbf{0 . 8 4 5 2}$ \\
FRFCM & $\boldsymbol{x}$ & 0.7397 & 0.0324 & $\mathbf{1}$ & 0.0482 \\
\hline REAT-RSSFCA & $\checkmark$ & $\mathbf{0 . 9 9 9 4}$ & $\mathbf{0 . 8 7 7 8}$ & 0.8702 & $\mathbf{0 . 8 6 7 3}$ \\
RSSFCA & $\boldsymbol{x}$ & 0.9057 & 0.0099 & $\mathbf{0 . 9 9 9 8}$ & 0.0189 \\
\hline
\end{tabular}




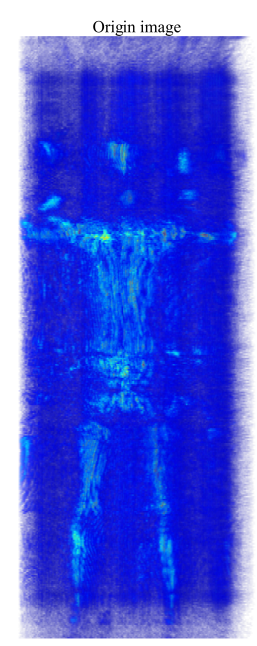

(a)

Ground truth

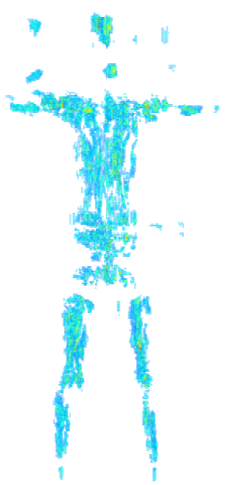

(f)

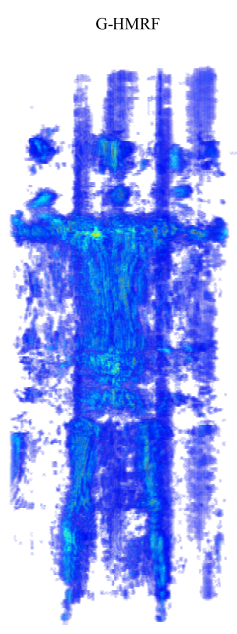

(b)

REAT-G-HMRF

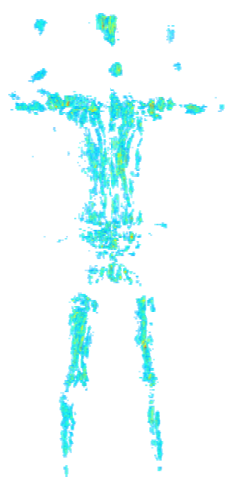

(g)

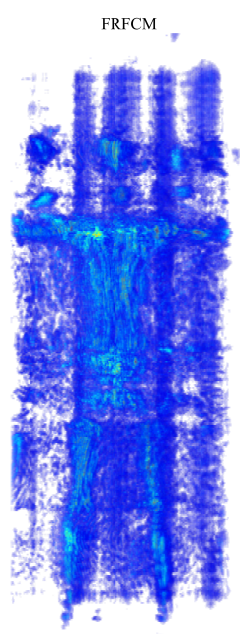

(c)

REAT-FRFCM

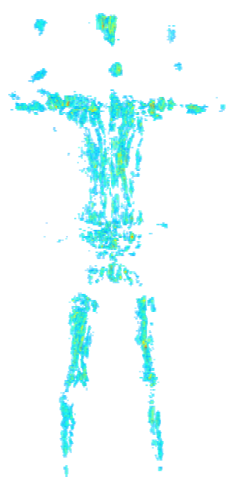

(h)

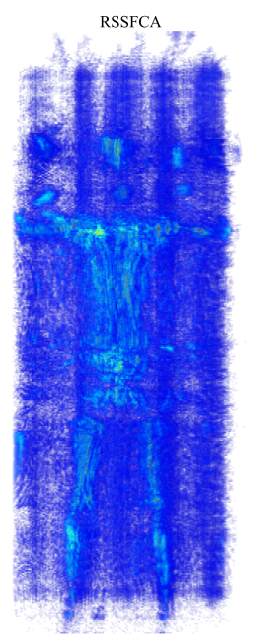

(d)

REAT-RSSFCA

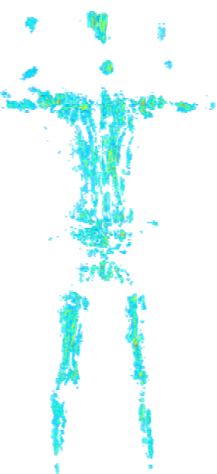

(i)
R-AMRFCM

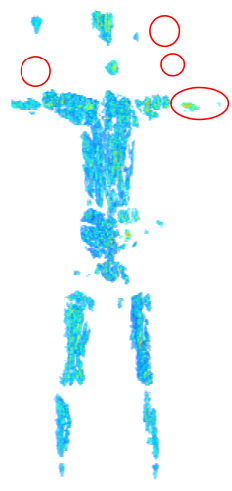

(e)

REAT-AMRFCM

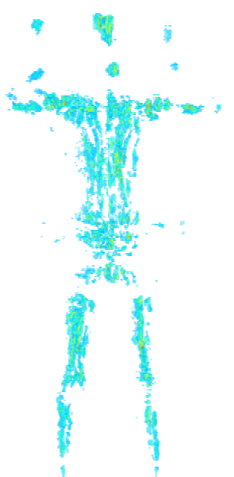

(j)

Figure 6. The original image, the ground truth, and the target extraction results of 8 algorithms. (a) Original image. (b) G-HMRF. (c) FRFCM. (d) RSSFCA. (e) R-AMRFCM. The areas where the target voxels are missing are marked by red circles. (f) Ground truth. (g) REAT-G-HMRF. (h) REAT-FRFCM. (i) REAT-RSSFCA. (j) REAT-AMRFCM. 


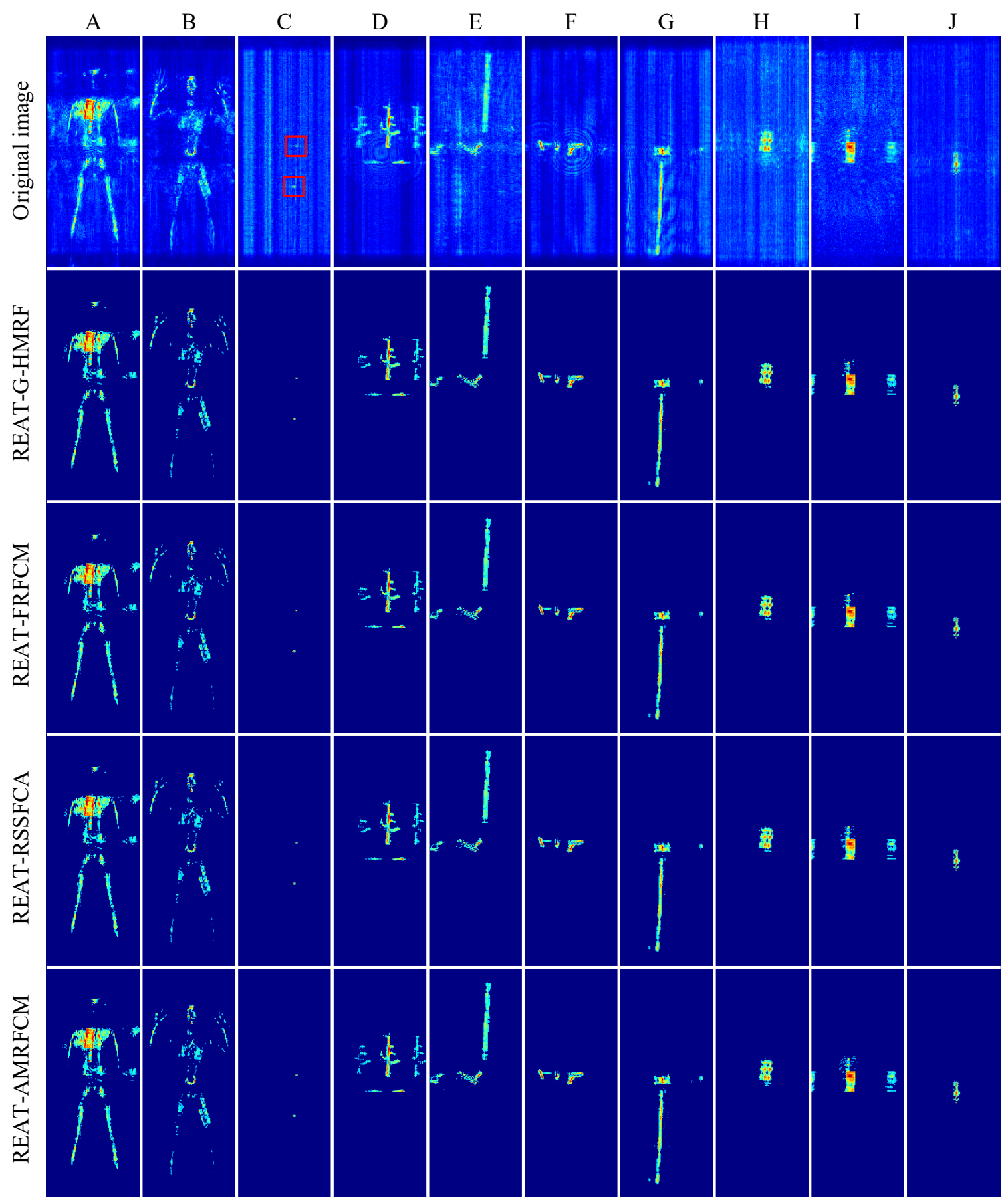

Figure 7. The original SAR images and the target extraction results of 10 scenes (Form (A) to (J)). The original images and the results are displayed in the maximum projection. Scene $(\mathbf{A})$ and $(\mathbf{B})$ : the human with kitchen knife. Scene (C): a tomato and a tiny metal ball (The targets are marked by the red boxes). Scene (D): a rifle and a knife (The rifle has two grating lobes and the knife has one grating lobe). Scene (E): a metal bar and a pistol. Scene (F): a knife and a pistol. Scene (G): a hammer. Scene (H): a mobile phone. Scene (I): a kitchen knife and its two grating lobes. Scene (J): a metal cup.

The evaluation results of REAT-AMRFCM and R-AMRFCM are shown in Table 2. The accuracy, precision, recall, and DSC of REAT-AMRFCM are 0.9994, 0.9170, 0.7920, and 0.8432 , respectively. The accuracy, precision, recall, and DSC of R-AMRFCM are 0.9988 , $0.7879,0.8187$, and 0.7792 , respectively. Compared with R-AMRFCM, REAT-AMRFCM improves the accuracy, the precision, and the DSC by 0.006, 0.1291 , and 0.0640 . The recall of REAT-AMRFCM is only 0.0267 lower than that of R-AMRFCM. DSC is the most important criterion among the four criteria, and the DSC of REAT-AMRFCM is significantly higher 
than that of R-AMRFCM. Hence, the performance of REAT-AMRFCM is higher than that of R-AMRFCM.

Table 2. Comparison of REAT-AMRFCM and R-AMRFCM. The bold number indicates the best performance under one criterion.

\begin{tabular}{ccccc}
\hline Method & Accuracy & Precision & Recall & DSC \\
\hline REAT-AMRFCM & $\mathbf{0 . 9 9 9 4}$ & $\mathbf{0 . 9 1 7 0}$ & 0.7920 & $\mathbf{0 . 8 4 3 2}$ \\
R-AMRFCM & 0.9988 & 0.7879 & $\mathbf{0 . 8 1 8 7}$ & 0.7792 \\
\hline
\end{tabular}

Consequently, the image analysis and evaluation results show that the proposed algorithm effectively enhances the anti-interference ability of the existing image segmentation methods. Moreover, the proposed algorithm has better performance and anti-interference ability than the ROI extraction algorithm in the state of the art.

\subsection{Analysis of Computational Efficiency}

Computational efficiency is an important evaluation criterion. Therefore, we test the time consumption of the above-mentioned algorithms to process the entire data set. The time consumption of the original algorithms and the enhanced algorithms are shown in Figure 8.

The orange bar represents the time consumption of the enhanced algorithms, and the blue bar represents the time consumption of the original algorithms. The time consumption of REAT-FRFCM is $12.96 \%$ lower than that of FRFCM. Although FRFCM has very high computational efficiency, the proposed algorithm still considerably improves the computational efficiency of FRFCM. The time consumption of REAT-G-HMRF is $96.64 \%$ lower than that of G-HMRF; thus, the proposed algorithm greatly improves the computational efficiency of G-HMRF. The time consumption of REAT-RSSFCA is $62.10 \%$ lower than that of RSSFCA, so the proposed algorithm also greatly improves the calculation efficiency of RSSFCA. Hence, the proposed algorithm causes a significant improvement in the calculation efficiency of the existing image segmentation methods.

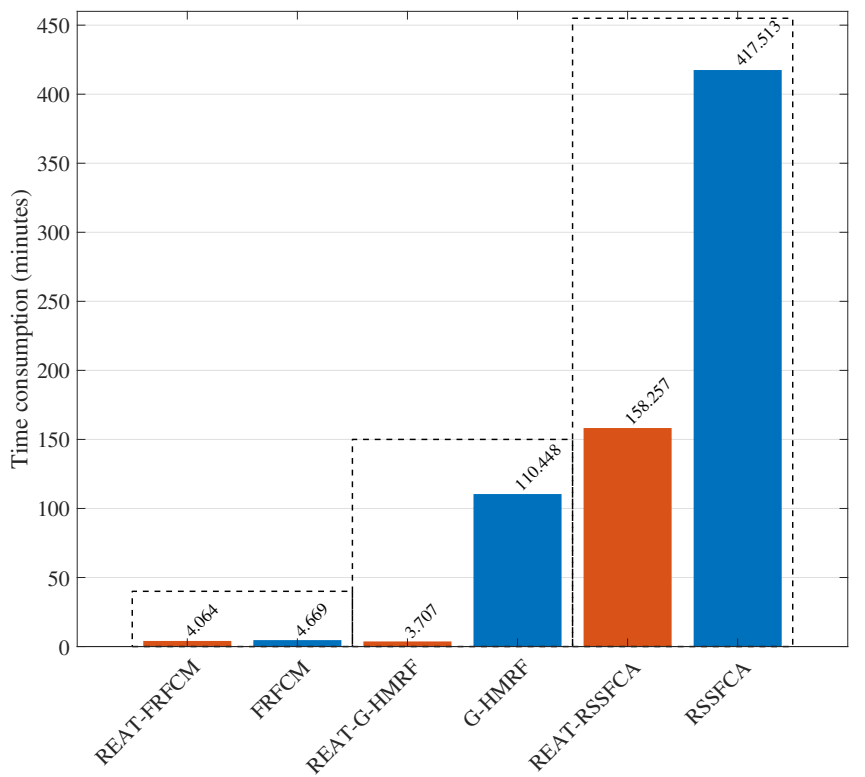

Figure 8. The time consumption of the six algorithms on the whole data set. The orange bar represents the time consumption of the enhanced algorithms. The blue bar represents the time consumption of the original algorithms.

As shown in Table 3, the time consumption of REAT-AMRFCM and R-AMRFCM are $9.2528 \mathrm{~min}$ and $11.8387 \mathrm{~min}$, respectively. The time consumption of REAT-AMRFCM 
is $21.84 \%$ lower than that of R-AMRFCM. Therefore, the proposed algorithm has higher computational efficiency than the ROI extraction algorithm in R-AMRFCM.

Table 3. The time consumption of REAT-AMRFCM and R-AMRFCM. The bold number indicates the best performance under one criterion.

\begin{tabular}{cc}
\hline Algorithm & Time Consumption (Minutes) \\
\hline REAT-AMRFCM & 9.2528 \\
R-AMRFCM & 11.8387 \\
\hline
\end{tabular}

In summary, the proposed algorithm not only enhances the performance of the existing image segmentation methods, but also enhances their computational efficiency. Moreover, the performance and the computational efficiency of the proposed algorithm are higher than those of the state-of-the-art algorithm.

\section{Discussion}

\subsection{Algorithm Visualization}

In this subsection, we show and discuss the results of several steps in the proposed algorithm. An image containing the rifle in the data set is used to illustrate the proposed algorithm. The 3-D SAR image with the size of $160 \times 400 \times 40$ is divided into 40 profiles with the size of $160 \times 400$ along the $\mathrm{Z}$ direction. The 16 profiles containing the target are tiled into a 2-D image, which is shown in Figure 9 . The saliency map, Figure $9 b$, shows that the saliency detection algorithm effectively highlights the target area in the 3-D SAR image. The original image and the saliency-enhanced image are shown in Figure $9 a, c$, respectively. As shown by the red boxes in these two figures, the saliency enhancement reduces the intensity of the background including interference. Figure $9 \mathrm{~d}$ is the image after anisotropic diffusion. Adaptive anisotropic diffusion filtering not only suppresses the noise but also makes the boundary between the target and the background clear, which is beneficial to the detection of the target edges. The extracted ROI is shown in Figure 9e. The core region is marked by white, the buffer area is marked by yellow, and the ground truth is marked by red. As shown in Figure 9e, the core region covers most of the target voxels. Due to inaccurate edge detection, some target voxels are lost. The buffer area effectively supplements the lost target voxels. Therefore, the extracted ROI almost completely covers the ground truth.

\subsection{The Integrity of the ROI}

In this subsection, we will discuss the integrity of the ROI extracted by the proposed algorithm and the role of the buffer area. The integrity of the ROI is defined as the ratio of the target voxels in the ROI to the ground truth. $\tau_{x}$ and $\tau_{y}$ have the same value, and their ranges are from 0 to 15 . Due to the small size in the $Z$ direction, $\tau_{z}$ is not discussed and set to $0 .\left(\tau_{x}, \tau_{y}, \tau_{z}\right)=(0,0,0)$ indicates that the buffer area is not working. In addition, the target extraction results of REAT-AMRFCM with different $\tau_{x}$ and $\tau_{y}$ are evaluated.

The evaluation results are shown in Table 4 and Figure 10. When the buffer area is not in effect, the integrity of the target region reaches $94.38 \%$ and the DSC is 0.7690 . When $\tau_{x}$ and $\tau_{y}$ are set to 15 , the integrity reaches $98.18 \%$ and the DSC is 0.8514 . As the size of the buffer area increases, the integrity and DSC continue to increase, and their growth rates continue to decrease. So, when $\tau_{x}$ and $\tau_{y}$ are large enough, the growth of the buffer area does not significantly improve the integrity. In summary, the introduction of the buffer area effectively supplements the target region, and the ROI extracted by the proposed algorithm almost completely covers the target region. 


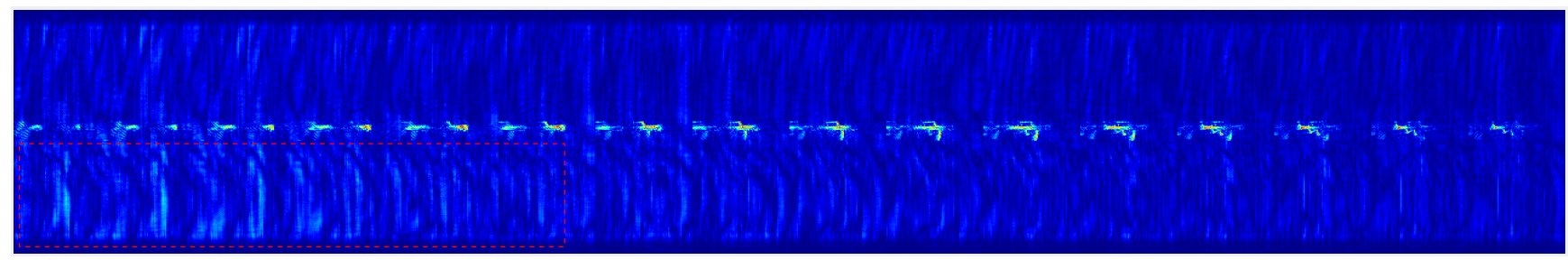

(a)

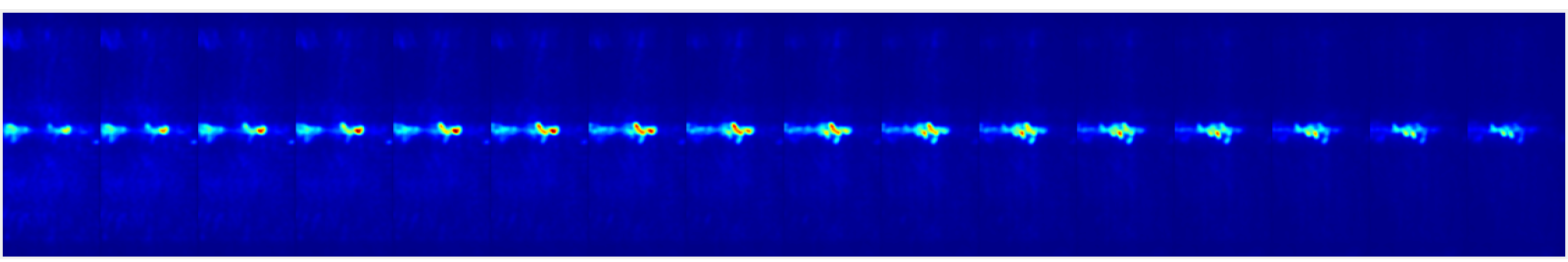

(b)

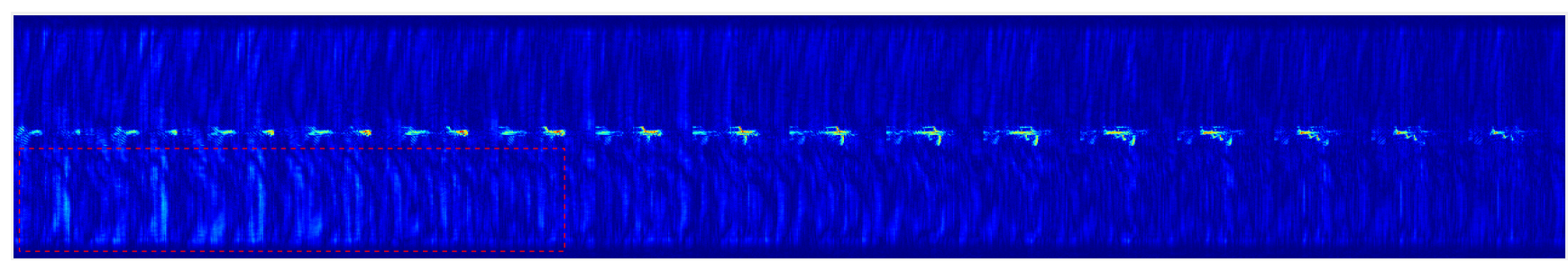

(c)

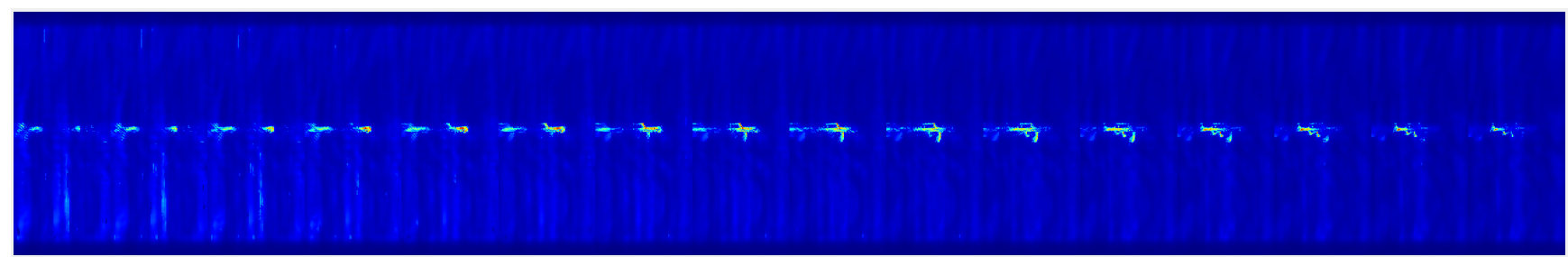

(d)

(e)

Figure 9. The illustration of the proposed algorithm. (a) The original image. (b) The saliency map. (c) The saliency enhanced image. (d) The image smoothed by the anisotropic diffusion method. (e) The extracted ROI. 


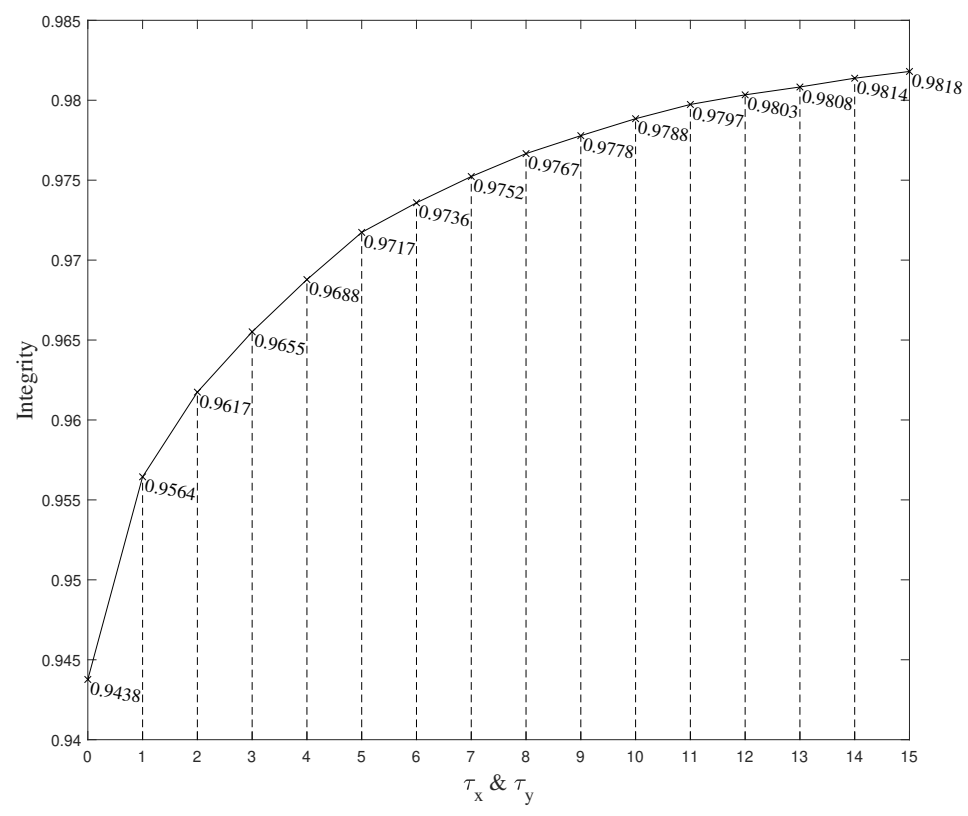

Figure 10. The relationship between the integrity of ROI extraction results and $\left(\tau_{x}, \tau_{y}\right)$.

Table 4 . The influence of $\left(\tau_{x}, \tau_{y}\right)$ on region integrity and performance.

\begin{tabular}{ccccccc}
\hline $\boldsymbol{\tau}$ & Integrity & Accuracy & Precision & Recall & DSC & Time (Minutes) \\
\hline 0 & 0.9438 & 0.9992 & 0.9550 & 0.6592 & 0.7690 & 8.2515 \\
1 & 0.9564 & 0.9993 & 0.9475 & 0.7015 & 0.7979 & 8.8571 \\
2 & 0.9617 & 0.9993 & 0.9386 & 0.7349 & 0.8176 & 9.0589 \\
3 & 0.9655 & 0.9994 & 0.9315 & 0.7571 & 0.8286 & 9.0809 \\
4 & 0.9688 & 0.9994 & 0.9247 & 0.7726 & 0.8349 & 9.3438 \\
5 & 0.9717 & 0.9994 & 0.9234 & 0.7814 & 0.8399 & 8.9220 \\
6 & 0.9736 & 0.9994 & 0.9167 & 0.7921 & 0.8431 & 9.2528 \\
7 & 0.9752 & 0.9994 & 0.9140 & 0.7979 & 0.8453 & 9.7222 \\
8 & 0.9767 & 0.9994 & 0.9105 & 0.8028 & 0.8463 & 10.7494 \\
9 & 0.9778 & 0.9994 & 0.9074 & 0.8074 & 0.8476 & 10.6927 \\
10 & 0.9788 & 0.9994 & 0.9024 & 0.8155 & 0.8491 & 11.4448 \\
11 & 0.9797 & 0.9994 & 0.9009 & 0.8181 & 0.8496 & 11.3717 \\
12 & 0.9803 & 0.9994 & 0.8977 & 0.8209 & 0.8493 & 12.2933 \\
13 & 0.9808 & 0.9994 & 0.8966 & 0.8242 & 0.8507 & 12.4019 \\
14 & 0.9814 & 0.9994 & 0.8948 & 0.8266 & 0.8509 & 13.4230 \\
15 & 0.9818 & 0.9994 & 0.8940 & 0.8284 & 0.8514 & 14.3674 \\
\hline
\end{tabular}

\section{Conclusions}

This paper proposed a flexible ROI extraction algorithm with adaptive threshold to enhance the anti-interference ability of existing image segmentation methods. The proposed algorithm utilized the saliency detection algorithm based on spectral residual to achieve image enhancement. The thresholds required by the anisotropic diffusion method and the Canny edge detection method were adaptively calculated by the features of the original image and the enhanced image. After the calculation of the thresholds, the anisotropic diffusion method and the Canny edge detection method were used to smooth the image and extract the target edges. The minimum bounding cuboids of the target edges detected by the Canny method were considered the core region. The boundary of the core region was expanded outward to obtain the complete ROI. The expanded buffer area reduced the loss of target regions caused by inaccurate edge extraction, which was verified in the discussion section. Moreover, the results of several steps were shown in the discussion section to illustrate their role. The proposed algorithm was utilized to enhance three existing image segmentation methods. The experimental results demonstrated that the proposed algorithm effectively enhances the anti-interference ability and the computational 
efficiency of the existing image segmentation methods. In addition, we replaced the ROI extraction part in R-AMRFCM with the proposed algorithm and named it REAT-AMRFCM. The performance and the computational efficiency of REAT-AMRFCM were better than those of R-AMRFCM.

For future work, we will explore image features more deeply to improve the antiinterference ability and the extraction accuracy of the target extraction algorithm.

Author Contributions: Conceptualization, L.L. and X.Z.; methodology, L.L. and C.W.; software, L.L. and B.T.; resources, L.P.; writing - review and editing, J.S. and S.W. All authors have read and agreed to the published version of the manuscript.

Funding: This work was supported in part by the National Key R\&D Program of China under Grant 2017YFB0502700, and in part by the National Natural Science Foundation of China under Grants 61571099,61501098 , and 61671113. All authors have read and agreed to the published version of the manuscript.

Institutional Review Board Statement: Not applicable.

Informed Consent Statement: Not applicable.

Data Availability Statement: Not applicable.

Acknowledgments: We thank anonymous reviewers for their comments towards improving this manuscript.

Conflicts of Interest: The authors declare no conflict of interest.

\section{References}

1. Ding, L.; Wu, S.; Li, P.; Zhu, Y. Millimeter-Wave Sparse Imaging for Concealed Objects Based on Sparse Range Migration Algorithm. IEEE Sens. J. 2019, 19, 6721-6728. [CrossRef]

2. Wang, C.; Shi, J.; Zhou, Z.; Li, L.; Zhou, Y.; Yang, X. Concealed Object Detection for Millimeter-wave Images with Normalized Accumulation Map. IEEE Sens. J. 2020, 21, 6468-6475. [CrossRef]

3. Liu, T.; Zhao, Y.; Wei, Y.; Zhao, Y.; Wei, S. Concealed object detection for activate millimeter wave image. IEEE Trans. Ind. Electron. 2019, 66, 9909-9917. [CrossRef]

4. Bi, H.; Tang, H.; Yang, G.; Shu, H.; Dillenseger, J.L. Accurate image segmentation using Gaussian mixture model with saliency map. Pattern Anal. Appl. 2018, 21, 869-878. [CrossRef]

5. Zhang, Y.; Brady, M.; Smith, S. Segmentation of brain MR images through a hidden Markov random field model and the expectation-maximization algorithm. IEEE Trans. Med Imaging 2001, 20, 45-57. [CrossRef]

6. Hassan, M.; Murtza, I.; Hira, A.; Ali, S.; Kifayat, K. Robust spatial fuzzy GMM based MRI segmentation and carotid artery plaque detection in ultrasound images. Comput. Methods Programs Biomed. 2019, 175, 179-192. [CrossRef] [PubMed]

7. Zhao, Q.h.; Li, X.1; Li, Y.; Zhao, X.m. A fuzzy clustering image segmentation algorithm based on hidden Markov random field models and Voronoi tessellation. Pattern Recognit. Lett. 2017, 85, 49-55. [CrossRef]

8. Zhang, X.; Ning, Y.; Li, X.; Zhang, C. Anti-noise FCM image segmentation method based on quadratic polynomial. Signal Process. 2021, 178, 107767. [CrossRef]

9. Tirandaz, Z.; Akbarizadeh, G.; Kaabi, H. PolSAR image segmentation based on feature extraction and data compression using weighted neighborhood filter bank and hidden Markov random field-expectation maximization. Measurement 2020, 153, 107432. [CrossRef]

10. Banerjee, A.; Maji, P. A spatially constrained probabilistic model for robust image segmentation. IEEE Trans. Image Process. 2020, 29, 4898-4910. [CrossRef] [PubMed]

11. Tang, Y.; Ren, F.; Pedrycz, W. Fuzzy C-means clustering through SSIM and patch for image segmentation. Appl. Soft Comput. 2020, 87, 105928. [CrossRef]

12. Tian, B.; Zhang, X.; Li, L.; Pu, L.; Pu, L.; Shi, J.; Wei, S. Fast Bayesian Compressed Sensing Algorithm via Relevance Vector Machine for LASAR 3D Imaging. Remote Sens. 2021, 13, 1751. [CrossRef]

13. Li, L.; Zhang, X.; Zhou, Y.; Pu, L.; Shi, J.; Wei, S. Region adaptive morphological reconstruction fuzzy C-means for near-field 3-D SAR image target extraction. Digit. Signal Process. 2021, 113, 103036. [CrossRef]

14. Gerig, G.; Kubler, O.; Kikinis, R.; Jolesz, F.A. Nonlinear anisotropic filtering of MRI data. IEEE Trans. Med Imaging 1992, 11, 221-232. [CrossRef]

15. Li, H.C.; Hong, W.; Wu, Y.R.; Fan, P.Z. An efficient and flexible statistical model based on generalized gamma distribution for amplitude SAR images. IEEE Trans. Geosci. Remote Sens. 2010, 48, 2711-2722.

16. Ziou, D.; Bouguila, N.; Allili, M.S.; El-Zaart, A. Finite gamma mixture modelling using minimum message length inference: Application to SAR image analysis. Int. J. Remote Sens. 2009, 30, 771-792. [CrossRef] 
17. Sportouche, H.; Nicolas, J.M.; Tupin, F. Mimic capacity of fisher and generalized gamma distributions for high-resolution SAR image statistical modeling. IEEE J. Sel. Top. Appl. Earth Obs. Remote Sens. 2017, 10, 5695-5711. [CrossRef]

18. Li, H.C.; Hong, W.; Wu, Y.R.; Fan, P.Z. On the empirical-statistical modeling of SAR images with generalized gamma distribution. IEEE J. Sel. Top. Signal Process. 2011, 5, 386-397.

19. Zhang, P.; Li, B.; Boudaren, M.E.Y.; Yan, J.; Li, M.; Wu, Y. Parameter Estimation of Generalized Gamma Distribution Toward SAR Image Processing. IEEE Trans. Aerosp. Electron. Syst. 2020, 56, 3701-3717. [CrossRef]

20. El-Zaart, A.; Ziou, D. Statistical modelling of multimodal SAR images. Int. J. Remote Sens. 2007, 28, 2277-2294. [CrossRef]

21. Cordeiro, G.M.; Cintra, R.J.; Rego, L.C.; Nascimento, A.D. The gamma generalized normal distribution: A descriptor of SAR imagery. J. Comput. Appl. Math. 2019, 347, 257-272. [CrossRef]

22. Li, H.C.; Krylov, V.A.; Fan, P.Z.; Zerubia, J.; Emery, W.J. Unsupervised learning of generalized gamma mixture model with application in statistical modeling of high-resolution SAR images. IEEE Trans. Geosci. Remote Sens. 2015, 54, 2153-2170. [CrossRef]

23. Wei, S.; Zhou, Z.; Wang, M.; Wei, J.; Liu, S.; Shi, J.; Zhang, X.; Fan, F. 3DRIED: A High-Resolution 3-D Millimeter-Wave Radar Dataset Dedicated to Imaging and Evaluation. Remote Sens. 2021, 13, 3366. [CrossRef]

24. Yanik, M.E.; Torlak, M. Near-field MIMO-SAR millimeter-wave imaging with sparsely sampled aperture data. IEEE Access 2019, 7, 31801-31819. [CrossRef]

25. Zou, H.; Qin, X.; Zhou, S.; Ji, K. A likelihood-based SLIC superpixel algorithm for SAR images using generalized Gamma distribution. Sensors 2016, 16, 1107. [CrossRef] [PubMed]

26. Dabboor, M.; Karathanassi, V.; Braun, A. A multi-level segmentation methodology for dual-polarized SAR data. Int. J. Appl. Earth Obs. Geoinf. 2011, 13, 376-385. [CrossRef]

27. Liu, H.; Wang, F.; Yang, S.; Hou, B.; Jiao, L.; Yang, R. Fast semisupervised classification using histogram-based density estimation for large-scale polarimetric SAR data. IEEE Geosci. Remote Sens. Lett. 2019, 16, 1844-1848. [CrossRef]

28. Bazi, Y.; Bruzzone, L.; Melgani, F. Automatic identification of the number and values of decision thresholds in the log-ratio image for change detection in SAR images. IEEE Geosci. Remote Sens. Lett. 2006, 3, 349-353. [CrossRef]

29. Qin, X.; Zou, H.; Zhou, S.; Ji, K. Region-based classification of SAR images using Kullback-Leibler distance between generalized gamma distributions. IEEE Geosci. Remote Sens. Lett. 2015, 12, 1655-1659.

30. Qin, X.; Zhou, S.; Zou, H. SAR image segmentation via hierarchical region merging and edge evolving with generalized gamma distribution. IEEE Geosci. Remote Sens. Lett. 2014, 11, 1742-1746.

31. Pappas, O.A.; Anantrasirichai, N.; Achim, A.M.; Adams, B.A. River planform extraction from high-resolution SAR images via generalized gamma distribution superpixel classification. IEEE Trans. Geosci. Remote Sens. 2020, 59, 3942-3955. [CrossRef]

32. Xiong, B.; Chen, J.M.; Kuang, G. A change detection measure based on a likelihood ratio and statistical properties of SAR intensity images. Remote Sens. Lett. 2012, 3, 267-275. [CrossRef]

33. Hou, X.; Zhang, L. Saliency detection: A spectral residual approach. In Proceedings of the 2007 IEEE Conference on Computer Vision and Pattern Recognition, Minneapolis, MN, USA, 17-22 June 2007; pp. 1-8.

34. Otsu, N. A threshold selection method from gray-level histograms. IEEE Trans. Syst. Man Cybern. 1979, 9, 62-66. [CrossRef]

35. Canny, J. A computational approach to edge detection. IEEE Trans. Pattern Anal. Mach. Intell. 1986, 8, 679-698. [CrossRef]

36. Geman, S.; Geman, D. Stochastic relaxation, Gibbs distributions, and the Bayesian restoration of images. IEEE Trans. Pattern Anal. Mach. Intell. 1984, 6, 721-741. [CrossRef] [PubMed]

37. Wang, Q. GMM-based hidden Markov random field for color image and 3D volume segmentation. arXiv 2012, arXiv:1212.4527.

38. Lei, T.; Jia, X.; Zhang, Y.; He, L.; Meng, H.; Nandi, A.K. Significantly fast and robust fuzzy c-means clustering algorithm based on morphological reconstruction and membership filtering. IEEE Trans. Fuzzy Syst. 2018, 26, 3027-3041. [CrossRef]

39. Jia, X.; Lei, T.; Du, X.; Liu, S.; Meng, H.; Nandi, A.K. Robust self-sparse fuzzy clustering for image segmentation. IEEE Access 2020, 8, 146182-146195. [CrossRef] 